\title{
Solving singular second-orderinitial/boundary value problems in reproducing kernel Hilbert space
}

\author{
Er Gao*, Songhe Song and Xinjian Zhang
}

\author{
* Correspondence: gao. \\ nudter@gmail.com \\ Department of Mathematics and \\ Systems Science, College of \\ Science, National University of \\ Defense Technology, Changsha \\ 410073, China
}

\begin{abstract}
In this paper, we presents a reproducing kernel method for computing singular second-order initial/boundary value problems (IBVPS). This method could deal with much more general IBVPs than the ones could do, which are given by the previous researchers. According to our work, in the first step, the analytical solution of IBVPS is represented in the RKHS which we constructs. Then, the analytic approximation is exhibited in this RKHS. Finally, the $n$-term approximation is proved to converge to the analytical solution. Some numerical examples are displayed to demonstrate the validity and applicability of the present method. The results obtained by using the method indicate the method is simple and effective.

Mathematics Subject Classification (2000) 35A24, 46E20, 47B32.
\end{abstract}

\section{Introduction}

Initial and boundary value problems of ordinary differential equations play an important role in many fields. Various applications of boundary to physical, biological, chemical, and other branches of applied mathematics are well documented in the literature. The main idea of this paper is to present a new algorithm for computing the solutions of singular second-order initial/boundary value problems (IBVPs) of the form:

$$
\left\{\begin{array}{l}
p(x) u^{\prime \prime}(x)+q(x) u^{\prime}(x)+r(x) u(x)=F(x, u) \\
a_{1} u(0)+b_{1} u^{\prime}(0)+c_{1} u(1)=0 \\
a_{2} u(1)+b_{2} u^{\prime}(1)+c_{2} u^{\prime}(0)=0
\end{array}\right.
$$

where $u(x) \in W_{2}^{3}[0,1]$, for $x \in[0,1], p \neq 0, p(x), q(x), r(x) \in \mathbf{C}[0,1] . a_{1}, b_{1}, c_{1}, a_{2}$, $b_{2}, c_{2}$ arc real constants and satisfy that $a_{1} u(0)+b_{1} u^{\prime}(0)+c_{1} u(1)$ and $a_{2} u(1)+$ $b_{2} u^{\prime}(1)+c_{2} u^{\prime}(0)$ are linear independent. $F(x, u)$ is continuous.

Remark 1.1. We find that if

$$
b_{1}=c_{1}=b_{2}=c_{2}=0, \quad a_{1} \neq 0, \quad a_{2} \neq 0,
$$

the problems are two-point BVPs; if

$$
b_{1}=c_{1}=a_{2}=b_{2}=0, \quad a_{1} \neq 0, \quad c_{2} \neq 0,
$$


the problems are initial value problems; if

$$
b_{1}=a_{2}=0, a_{1}=c_{1} \neq 0, b_{2}=c_{2} \neq 0,
$$

the problems are periodic BVPs; if

$$
b_{1}=a_{2}=0, \quad a_{1}=-c_{1} \neq 0, \quad b_{2}=-c_{2} \neq 0,
$$

the problems are anti-periodic BVPs.

Such problems have been investigated in many researches. Specially, the existence and uniqueness of the solution of (1.1) have been discussed in [1-5]. And in recent years, there are also a large number of special-purpose methods are proposed to provide accurate numerical solutions of the special form of (1.1), such as collocation methods [6], finite-element methods [7], Galerkin-wavelet methods [8], variational iteration method [9], spectral methods [10], finite difference methods [11], etc.

On the other hands, reproducing kernel theory has important applications in numerical analysis, differential equation, probability and statistics, machine learning and precessing image. Recently, using the reproducing kernel method, Cui and Geng [12-16] have make much effort to solve some special boundary value problems.

According to our method, which is presented in this paper, some reproducing kernel Hilbert spaces have been presented in the first step. And in the second step, the homogeneous IBVPs is deal with in the RKHS. Finally, one analytic approximation of the solutions of the second-order BVPs is given by reproducing kernel method under the assumption that the solution to (1.1) is unique.

\section{Some RKHS}

In this section, we will introduce the RKHS $W_{2}^{1}[0,1]$ and $W_{2}^{3}[0,1]$. Then we will construct a RKHS $H_{2}^{3}[0,1]$, in which every function satisfies the boundary condition of (1.1).

\subsection{The RKHS $W_{2}^{1}[0,1]$}

Inner space $W_{2}^{1}[0,1]$ is defined as $W_{2}^{1}[0,1]=\{u(x) \mid u$ is absolutely continuous real valued functions, $\left.u^{\prime} \in L^{2}[0,1]\right\}$. The inner product in $W_{2}^{1}[0,1]$ is given by

$$
(f, h)_{W_{2}^{1}}=f(0) h(0)+\int_{0}^{1} f^{\prime}(t) h^{\prime}(t) \mathrm{d} t, \quad f, h \in W_{2}^{1}[0,1]
$$

and the norm $\|u\|_{W_{2}^{1}}$ is denoted by $\|u\|_{W_{2}^{1}}=\sqrt{(u, u)_{W_{2}^{1}}}$. From $[17,18], W_{2}^{1}[0,1]$ is a reproducing kernel Hilbert space and the reproducing kernel is

$$
K_{1}(t, s)=1+\min \{t, s\}
$$

\subsection{The RKHS $W_{2}^{3}[0,1]$}

Inner space $W_{2}^{3}[0,1]$ is defined as $W_{2}^{3}[0,1]=\left\{u(x) \mid u, u^{\prime}, u^{\prime \prime}\right.$ is absolutely continuous real valued functions, $\left.u^{\prime \prime \prime} \in L^{2}[0,1]\right\}$.

From [15,17-19], it is clear that $W_{2}^{3}[0,1]$ become a reproducing kernel Hilbert space if we endow it with suitable inner product. 
Zhang and Lu [18] and Long and Zhang [19] give us a clue to relate the inner product with the boundary conditions (1.1). Set $L=D^{3}$, and

$$
\left\{\begin{array}{l}
\gamma_{1} f=a_{1} f(0)+b_{1} f^{\prime}(0)+c_{1} f(1) \\
\gamma_{2} f=a_{2} f(1)+b_{2} f^{\prime}(1)+c_{2} f^{\prime}(0) \\
\gamma_{3} f=a_{3} f(0)+b_{3} f^{\prime}(0)+c_{3} f^{\prime \prime}(0)
\end{array}\right.
$$

where $a_{3}, b_{3}, c_{3}$ is random but satisfying that $\gamma_{3}$ is linearly independent of $\gamma_{1}$ and $\gamma_{2}$. It is easy to know that $\gamma_{1}, \gamma_{2}, \gamma_{3}$ are linearly independent in $\operatorname{Ker} L$. Then from [18,19], it is easy to know one of the inner products of $W_{2}^{3}[0,1]$

$$
(f, h)_{W_{2}^{3}}=\sum_{i=1}^{3} \gamma_{i} f \gamma_{i} h+\int_{0}^{1} f^{\prime \prime \prime}(t) h^{\prime \prime \prime}(t) \mathrm{d} t, \quad f, h \in W_{2}^{3}[0,1]
$$

and its corresponding reproducing kernel $K_{2}(t, s)$.

\subsection{The RKHS $H_{2}^{3}[0,1]$}

Inner space $H_{2}^{3}[0,1]$ is defined as $H_{2}^{3}[0,1]=\left\{u(x) \mid u, u^{\prime}, u^{\prime \prime}\right.$ are absolutely continuous real valued functions, $u^{\prime \prime \prime} \in L^{2}[0,1]$, and, $a_{1} u(0)+b_{1} u^{\prime}(0)+c_{1} u(1)=0, a_{2} u(1)+$ $\left.b_{2} u^{\prime}(1)+c_{2} u^{\prime}(0)=0\right\}$.

It is clear that $H_{2}^{3}[0,1]$ is the complete subspace of $W_{2}^{3}[0,1]$, so $H_{2}^{3}[0,1]$ is a RKHS. If $P$, which is the orthogonal projection from $W_{2}^{3}[0,1]$ to $H_{2}^{3}[0,1]$, is found, we can get the reproducing kernel of $H_{2}^{3}[0,1]$ obviously. Under the assumptions of Section 2, note

$$
P f(t)=\left(\gamma_{3} f\right) e_{3}(t)+\int_{0}^{1} G(t, \tau) \cdot f^{\prime \prime \prime}(\tau) \mathrm{d} \tau, \quad \forall f \in W_{2}^{3}[0,1]
$$

Theorem 2.1. Under the assumptions above, $P$ is the orthogonal projection from $H_{2}^{3}[0,1]$ to $H_{2}^{3}[0,1]$.

Proof. For all $f \in W_{2}^{3}[0,1]$, We have

$$
\left(\gamma_{1}(P f)\right)(t)=\left(\gamma_{2}(P f)\right)(t)=0
$$

That means $P f \in H_{2}^{3}[0,1]$. At the same time, for any $f, h \in W_{2}^{3}[0,1]$

$$
\begin{aligned}
(P f, h) & =\left(\left(\gamma_{3} f\right) e_{3}(t)+\int_{0}^{1} G(t, \tau) \cdot L f(\tau) \mathrm{d} \tau, h\right) \\
& =\left(\gamma_{3} f\right)\left(\gamma_{3} h\right)+\int_{0}^{1}\left(L \int_{0}^{1} G(t, \tau) \cdot L f(\tau) d \tau\right) \cdot L h(t) \mathrm{d} t \\
& =\left(\gamma_{3} f\right)\left(\gamma_{3} h\right)+\int_{0}^{1} L f(t) \cdot \operatorname{Lh}(t) \mathrm{d} t \\
(f, P h) & =\left(f,\left(\gamma_{3} h\right) e_{3}(t)+\int_{0}^{1} G(t, \tau) \cdot \operatorname{Lh}(\tau) \mathrm{d} \tau\right) \\
& =\left(\gamma_{3} f\right)\left(\gamma_{3} h\right)+\int_{0}^{1} L f(t) \cdot L \int_{0}^{1} G(t, \tau) \cdot \operatorname{Lh}(\tau) \mathrm{d} \tau \mathrm{d} t \\
& =\left(\gamma_{3} f\right)\left(\gamma_{3} h\right)+\int_{0}^{1} L f(t) \cdot \operatorname{Lh}(t) \mathrm{d} t
\end{aligned}
$$


$P$ is self-conjugate. And

$$
\begin{aligned}
P(P f) & =P\left(\left(\gamma_{3} f\right) e_{3}(t)+\int_{0}^{1} G(t, \tau) \cdot L f(\tau) \mathrm{d} \tau\right) \\
& =\left(\gamma_{3} f\right) e_{3}(t)+\int_{0}^{1} G(t, \tau) \cdot L\left(\left(\gamma_{3} f\right) e_{3}(\tau)+\int_{0}^{1} G(\tau, s) \cdot L f(s) \mathrm{d} s\right), \mathrm{d} \tau \\
& =\left(\gamma_{3} f\right) e_{3}(t)+\int_{0}^{1} G(t, \tau) \cdot L f(\tau) \mathrm{d} \tau \\
& =P f
\end{aligned}
$$

$P$ is idempotent.

So $P$ is the orthogonal projection from $W_{2}^{3}[0,1]$ to $H_{2}^{3}[0,1]$.

The proof of the Theorem 2.1 is complete.

Now, $H_{2}^{3}[0,1]$ is a RKHS if endowed the inner product with the inner product below

$$
(f, h)_{H_{2}^{3}}=\gamma_{3} f \gamma_{3} h+\int_{0}^{1} f^{\prime \prime \prime}(t) \cdot h^{\prime \prime \prime}(t) \mathrm{d} t
$$

and the corresponding reproducing kernel $K_{3}(t, s)$ is given in Appendix 4.

\section{The reproducing kernel method}

In this section, the representation of analytical solution of (1.1) is given in the reproducing kernel space $H_{2}^{3}[0,1]$.

Note $L u=p(x) u^{\prime \prime}(x)+q(x) u^{\prime}(x)+r(x) u(x)$ in (1.1). It is clear that $L: H_{2}^{3}[0,1] \rightarrow W_{2}^{1}[0,1]$ is a bounded linear operator.

Put $\phi_{i}(x)=K_{1}\left(x_{i}, x\right), \Psi_{i}(x)=L^{*} \phi_{i}(x)$, where $L^{*}$ is the adjoint operator of $L$. Then

$$
\begin{aligned}
\Psi_{i}(x) & =\left(L * \varphi_{i}(y), K_{3}(x, y)\right) \\
& =\left(\varphi_{i}(y), L_{\gamma} K_{3}(x, y)\right) \\
& =\overline{\left(L_{\gamma} K_{3}(x, y), \varphi_{i}(x)\right)}=\left.L_{\gamma} K_{3}(x, y)\right|_{y=x_{i}}
\end{aligned}
$$

Lemma 3.1. Under the assumptions above, if $\left\{x_{i}\right\}_{i=1}^{\infty}$ is dense on $[0,1]$ then $\left\{\Psi_{i}(x)\right\}_{i=1}^{\infty}$ is the complete basis $H_{2}^{3}[0,1]$.

The orthogonal system $\left\{\overline{\Psi_{i}}(x)\right\}_{i=1}^{\infty}$ of $H_{2}^{3}[0,1]$ can be derived from Gram-Schmidt orthogonalization process of $\left\{\Psi_{i}(x)\right\}_{i=1}^{\infty}$, and

$$
\overline{\Psi_{i}}(x)=\sum_{j=1}^{i} \beta_{i j} \Psi_{j}(x) .
$$

Then

Theorem 3.1. If $\left\{x_{i}\right\}_{i=1}^{\infty}$ is dense on $[0,1]$ and the solution of $(1.1)$ is unique, the solution can be expressed in the form

$$
u(x)=\sum_{i=1}^{\infty} \sum_{k=1}^{i} \beta_{i k} F\left(x_{k}, u\left(x_{k}\right)\right) \Psi_{i}(x)
$$


Proof. From Lemma 3.1, $\left\{\Psi_{i}(x)\right\}_{i=1}^{\infty}$ is the complete system of $H_{2}^{3}[0,1]$. Hence we have

$$
\begin{aligned}
u(x) & =\sum_{i=1}^{\infty}\left(u(x), \bar{\Psi}_{i}(x)\right) \bar{\Psi}_{i}(x)=\sum_{i=1}^{\infty} \sum_{k=1}^{i} \beta_{i k}\left(u(x), \Psi_{i}(x)\right) \bar{\Psi}_{i}(x) \\
& =\sum_{i=1}^{\infty} \sum_{k=1}^{i} \beta_{i k}\left(u(x), L * \varphi_{k}(x)\right) \bar{\Psi}_{i}(x)=\sum_{i=1}^{\infty} \sum_{k=1}^{i} \beta_{i k}\left(L u(x), \varphi_{k}(x)\right) \bar{\Psi}_{i}(x) \\
& =\sum_{i=1}^{\infty} \sum_{k=1}^{i} \beta_{i k}\left(F(x, u(x)), \varphi_{k}(x)\right) \bar{\Psi}_{i}(x)=\sum_{i=1}^{\infty} \sum_{k=1}^{i} \beta_{i k} F\left(x_{k}, u\left(x_{k}\right)\right) \bar{\Psi}_{i}(x)
\end{aligned}
$$

and the proof is complete.

The approximate solution of the (1.1) is

$$
u_{n}(x)=\sum_{i=1}^{n} \sum_{k=1}^{i} \beta_{i k} F\left(x_{k}, u\left(x_{k}\right)\right) \bar{\Psi}_{i}(x)
$$

If (1.1) is linear, that is $F(x, u(x))=F(x)$, then the approximate solution of (1.1) can be obtained directly from (3.3). Else, the approximate process could be modified into the following form:

$$
\left\{\begin{array}{l}
u_{0}(x)=0 \\
u_{n+1}(x)=\sum_{i=1}^{n+1} B_{i} \bar{\Psi}_{i}(x)
\end{array}\right.
$$

where $B_{i}=\sum_{k=1}^{i} \beta_{i k} F\left(x_{k}, u_{n}\left(x_{k}\right)\right)$.

Next, the convergence of $u_{n}(x)$ will be proved.

Lemma 3.2. There exists a constant $M$, satisfied $|u(x)| \leq M|| u \|_{H_{2}^{3}}$, for all $u(x) \in H_{2}^{3}[0,1]$.

Proof. For all $x \in[0,1]$ and $u \in H_{2}^{3}[0,1]$, there are

$$
|u(x)|=\left|\left(u(\cdot), K_{3}(\cdot, x)\right)\right| \leq\left\|K_{3}(\cdot, x)\right\|_{H_{2}^{3}} \cdot\|u\|_{H_{2}^{3}} .
$$

Since $K_{3}(\cdot, x) \in H_{2}^{3}[0,1]$, note

$$
M=\max _{x \in[0,1]}\left\|K_{3}(\cdot, x)\right\|_{H_{2}^{3}} .
$$

That is,

$$
|u(x)| \leq\left. M|| u\right|_{H_{2}^{3}}
$$

By Lemma 3.2, it is easy to obtain the following lemma.

Lemma 3.3. If $u_{n} \stackrel{\|\cdot\|}{\rightarrow} \bar{u}(n \rightarrow \infty),\left\|u_{n}\right\|$ is bounded, $x_{n} \rightarrow y(n \rightarrow \infty)$ and $F(x, u(x))$ is continuous, then $F\left(x_{n}, u_{n-1}\left(x_{n}\right)\right) \rightarrow F(y, \bar{u}(y))$.

Theorem 3.2. Suppose that $\left\|u_{n}\right\|$ is bounded in (3.3) and (1.1) has a unique solution. If $\left\{x_{i}\right\}_{i=1}^{\infty}$ is dense on $[0,1]$, then the $n$-term approximate solution $u_{n}(x)$ derived from the above method converges to the analytical solution $u(x)$ of (1.1).

Proof. First, we will prove the convergence of $u_{n}(x)$. 
From (3.4), we infer that

$$
u_{n+1}(x)=u_{n}(x)+B_{n+1} \bar{\Psi}_{n+1}(x) .
$$

The orthonormality of $\left\{\bar{\Psi}_{i}\right\}_{i=1}^{\infty}$ yield that

$$
\left\|u_{n+1}\right\|^{2}=\left\|u_{n}\right\|^{2}+\left(B_{n+1}\right)^{2}=\cdots=\sum_{i=1}^{n+1}\left(B_{i}\right)^{2}
$$

That means $\left\|u_{n+1}\right\| \geq\left\|u_{n}\right\|$. Due to the condition that $\left\|u_{n}\right\|$ is bounded, $\left\|u_{n}\right\|$ is convergent and there exists a constant $\ell$ such that

$$
\sum_{i=1}^{\infty}\left(B_{i}\right)^{2}=\ell
$$

If $m>n$, then

$$
\left\|u_{m}-u_{n}\right\|^{2}=\left\|u_{m}-u_{m-1}+u_{m-1}-u_{m-2}+\cdots+u_{n+1}-u_{n}\right\|^{2} .
$$

In view of $\left(u_{m}-u_{m-1}\right) \perp\left(u_{m-1}-u_{\mathrm{m}-2}\right) \perp \cdots \perp\left(u_{n+1}-u_{n}\right)$, it follows that

$$
\begin{aligned}
\left\|u_{m}-u_{n}\right\|^{2} & =\left\|u_{m}-u_{m-1}\right\|^{2}+\left\|u_{m-1}-u_{m-2}\right\|^{2}+\cdots+\left\|u_{n+1}-u_{n}\right\|^{2} \\
& =\sum_{i=n+1}^{m}\left(B_{i}\right)^{2} \rightarrow 0 \quad \text { as } \quad n \rightarrow \infty
\end{aligned}
$$

The completeness of $H_{2}^{3}[0,1]$ shows that $u_{n} \rightarrow \bar{u}$ as $n \rightarrow \infty$ in the sense of $\|\cdot\|_{H_{2}^{3}}$. Secondly, we will prove that $\bar{u}$ is the solution of (1.1).

Taking limits in (3.2), we get

$$
\bar{u}(x)=\sum_{i=1}^{\infty} B_{i} \bar{\Psi}_{i}(x) .
$$

So

$$
L \bar{u}(x)=\sum_{i=1}^{\infty} B_{i} L \bar{\Psi}_{i}(x)
$$

and

$$
(L \bar{u})(x)=\sum_{i=1}^{\infty} B_{i}\left(L \bar{\Psi}_{i}, \varphi_{n}\right)=\sum_{i=1}^{\infty} B_{i}\left(\bar{\Psi}_{i}, L * \varphi_{n}\right)=\sum_{i=1}^{\infty} B_{i}\left(\bar{\Psi}_{i}, \Psi_{n}\right) .
$$

Therefore,

$$
\sum_{i=1}^{n} \beta_{n j}(L \bar{v})\left(x_{n}\right)=\sum_{i=1}^{\infty} B_{i}\left(\bar{\Psi}_{i}, \sum_{i=1}^{n} \beta_{n j} \Psi_{j}\right)=\sum_{i=1}^{\infty} B_{i}\left(\bar{\Psi}_{i}, \bar{\Psi}_{n}\right)=B_{n} .
$$

If $n=1$, then

$$
\operatorname{Lu}\left(x_{1}\right)=F\left(x_{1}, u_{0}\left(x_{1}\right)\right) .
$$

If $n=2$, then

$$
\beta_{21} L \bar{u}\left(x_{1}\right)+\beta_{22} L \bar{u}\left(x_{2}\right)=\beta_{21} F\left(x_{1}, u_{0}\left(x_{1}\right)\right)+\beta_{22} F\left(x_{2}, u_{1}\left(x_{2}\right)\right) .
$$


It is clear that

$$
(L \bar{u})\left(x_{2}\right)=F\left(x_{2}, u_{1}\left(x_{2}\right)\right) .
$$

Moreover, it is easy to see by induction that

$$
(L \bar{u})\left(x_{j}\right)=F\left(x_{j}, u_{j-1}\left(x_{j}\right)\right), \quad j=1,2, \ldots
$$

Since $\left\{x_{i}\right\}_{i=1}^{\infty}$ is dense on $[0,1]$, for all $Y \in[0,1]$, there exists a subsequence $\left\{x_{n j}\right\}_{j=1}^{\infty}$ such that

$$
x_{n j} \rightarrow Y \quad \text { as } \quad j \rightarrow \infty .
$$

It is easy to see that $(L \bar{u})\left(x_{n j}\right)=F\left(x_{n j}, u_{n j-1}\left(x_{n j}\right)\right)$. Let $j \rightarrow \infty$, by the continuity of $F(x$, $u(x))$ and Lemma 3.3, we have

$$
(L \bar{u})(Y)=F(Y, \bar{u}(Y))
$$

At the same time, $\bar{u} \in H_{2}^{3}[0,1]$. Clearly, $u$ satisfies the boundary conditions of (1.1).

That is, $\bar{u}$ is the solution of (1.1).

The proof is complete.

In fact, $u_{n}(x)$ is just the orthogonal projection of exact solution $\bar{u}(x)$ onto the space $\overline{\operatorname{Span}\left\{\bar{\Psi}_{i}\right\}_{i=1}^{n}}$.

\section{Numerical example}

In this section, some examples are studied to demonstrate the validity and applicability of the present method. We compute them and compare the results with the exact solution of each example.

Example 4.1. Consider the following IBVPs:

$$
\left\{\begin{array}{l}
x^{2}(1-x) u^{\prime \prime}(x)+2 u^{\prime}(x)+10 x u(x)+x^{2}(1-x)(u(x)+1)^{2}=f(x), 0<x<1, \\
u(0)+u^{\prime}(0)+u(1)=0, \\
u(1)+u^{\prime}(1)+u^{\prime}(0)=1,
\end{array}\right.
$$

$f(x)=10 x e^{10\left(x-x^{2}\right)^{2}}+40 e^{10\left(x-x^{2}\right)^{2}(1-2 x)\left(x-x^{2}\right)}+x^{2}(1-x)\left(e^{20\left(x-x^{2}\right)^{2}}+20 e^{10\left(x-x^{2}\right)^{2}}(1-2 x)^{2}-\right.$
Where
$\left.40 e^{10\left(x-x^{2}\right)^{2}}\left(x-x^{2}\right)+400 e^{10\left(x-x^{2}\right)^{2}}(1-2 x)^{2}\left(x-x^{2}\right)^{2}\right)$ .

The exact solution is $u(x)=e^{10\left(x-x^{2}\right)^{2}}-1$. Using our method, take $a_{3}=1, b_{3}=c_{3}=0$ and $n=21,51, N=5, x_{i}=\frac{i-1}{n-1}$. The numerical results are given in Tables 1 and 2 .

Example 4.2. Consider the following IBVPs:

$$
\left\{\begin{array}{l}
u^{\prime \prime}(x)+u^{\prime}(x)+x(1-x)(u(x)-1)^{3}=f(x), 0 \leq x \leq 1 \\
-\frac{\pi}{2} u(0)+u^{\prime}(0)-\frac{\pi}{2} u(1)=0 \\
\pi u(1)+2 u^{\prime}(1)+3 u^{\prime}(0)=0
\end{array}\right.
$$

where $f(x)=\pi \cos (\pi x)-\sin (\pi x)\left(x^{2}+(-1+x) * x * \sin ^{2}\left(\pi^{*} x\right)\right)$. The true solution is $u$ $(x)=\sin (\pi x)+1$. Using our method, take $a_{3}=1, b_{3}=\mathrm{c}_{3}=0$, and $N=5, n=21,51$, $x_{i}=\frac{i-1}{n-1}$. The numerical results are given in Figures $1,2,3$, and 4 . 
Table 1 Numerical results for Example $4.1(n=21, N=5)$

\begin{tabular}{lllll}
\hline $\boldsymbol{x}$ & True solution $\boldsymbol{u}(\boldsymbol{x})$ & Approximate solution $\boldsymbol{u}_{\mathbf{1 1}}$ & Absolute error & Relative error \\
\hline 0.08 & 0.05566 & 0.05530 & $3.6 \mathrm{E}-4$ & $6.5 \mathrm{E}-3$ \\
0.16 & 0.19798 & 0.19765 & $3.3 \mathrm{E}-4$ & $1.7 \mathrm{E}-3$ \\
0.24 & 0.39473 & 0.39443 & $3.0 \mathrm{E}-4$ & $7.6 \mathrm{E}-4$ \\
0.32 & 0.60560 & 0.60526 & $3.4 \mathrm{E}-4$ & $5.6 \mathrm{E}-4$ \\
0.40 & 0.77891 & 0.77839 & $5.2 \mathrm{E}-4$ & $6.6 \mathrm{E}-4$ \\
0.48 & 0.86452 & 0.86385 & $6.7 \mathrm{E}-4$ & $7.7 \mathrm{E}-4$ \\
0.56 & 0.83516 & 0.83457 & $5.9 \mathrm{E}-4$ & $7.1 \mathrm{E}-4$ \\
0.64 & 0.70036 & 0.70009 & $2.7 \mathrm{E}-4$ & $3.8 \mathrm{E}-4$ \\
0.72 & 0.50144 & 0.50146 & $1.8 \mathrm{E}-5$ & $3.6 \mathrm{E}-4$ \\
0.80 & 0.29175 & 0.29175 & $3.2 \mathrm{E}-6$ & $1.1 \mathrm{E}-5$ \\
0.88 & 0.11797 & 0.11771 & $2.6 \mathrm{E}-4$ & $2.2 \mathrm{E}-3$ \\
0.96 & 0.01485 & 0.01453 & $3.3 \mathrm{E}-4$ & $2.2 \mathrm{E}-3$ \\
\hline
\end{tabular}

Table 2 Numerical results for Example $4.1(n=51, N=5)$

\begin{tabular}{lllll}
\hline $\boldsymbol{x}$ & True solution $\boldsymbol{u}(\boldsymbol{x})$ & Approximate solution $\boldsymbol{u}_{\mathbf{1 1}}$ & Absolute error & Relative error \\
\hline 0.08 & 0.05566 & 0.05564 & $2.3 \mathrm{E}-5$ & $4 . \mathrm{EE}-4$ \\
0.16 & 0.19798 & 0.19796 & $2 . \mathrm{E}-5$ & $1.1 \mathrm{E}-4$ \\
0.24 & 0.39473 & 0.39471 & $2.0 \mathrm{E}-5$ & $4.9 \mathrm{E}-5$ \\
0.32 & 0.60560 & 0.60557 & $2.8 \mathrm{E}-5$ & $4.6 \mathrm{E}-5$ \\
0.40 & 0.77891 & 0.77885 & $5.6 \mathrm{E}-5$ & $7.5 \mathrm{E}-5$ \\
0.48 & 0.86452 & 0.86444 & $8.0 \mathrm{E}-5$ & $9.3 \mathrm{E}-5$ \\
0.56 & 0.83516 & 0.83509 & $6.6 \mathrm{E}-5$ & $7.9 \mathrm{E}-5$ \\
0.64 & 0.70036 & 0.70035 & $9.6 \mathrm{E}-6$ & $1.4 \mathrm{E}-5$ \\
0.72 & 0.50144 & 0.50148 & $4.3 \mathrm{E}-5$ & $8.6 \mathrm{E}-5$ \\
0.80 & 0.29175 & 0.29180 & $4.7 \mathrm{E}-5$ & $1.6 \mathrm{E}-5$ \\
0.88 & 0.11797 & 0.11797 & $2.2 \mathrm{E}-6$ & $1.9 \mathrm{E}-5$ \\
\hline
\end{tabular}

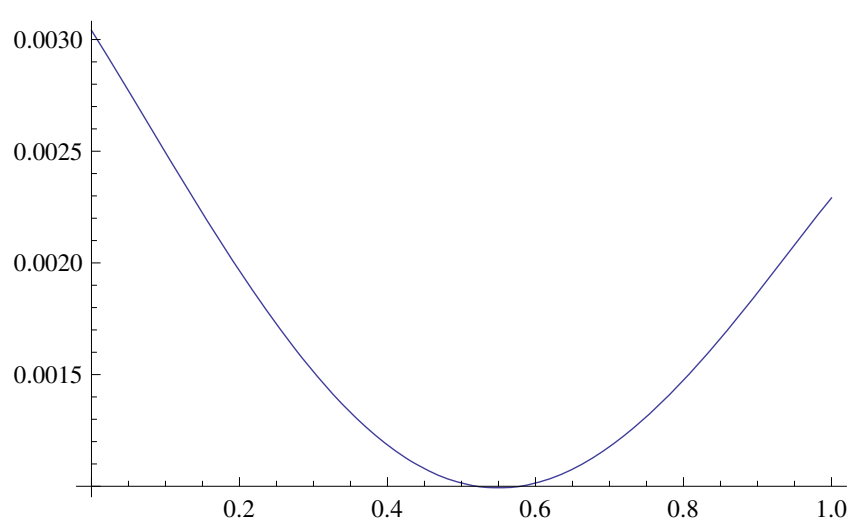

Figure 1 The absolute error of Example $4.2(n=21, N=5)$. 

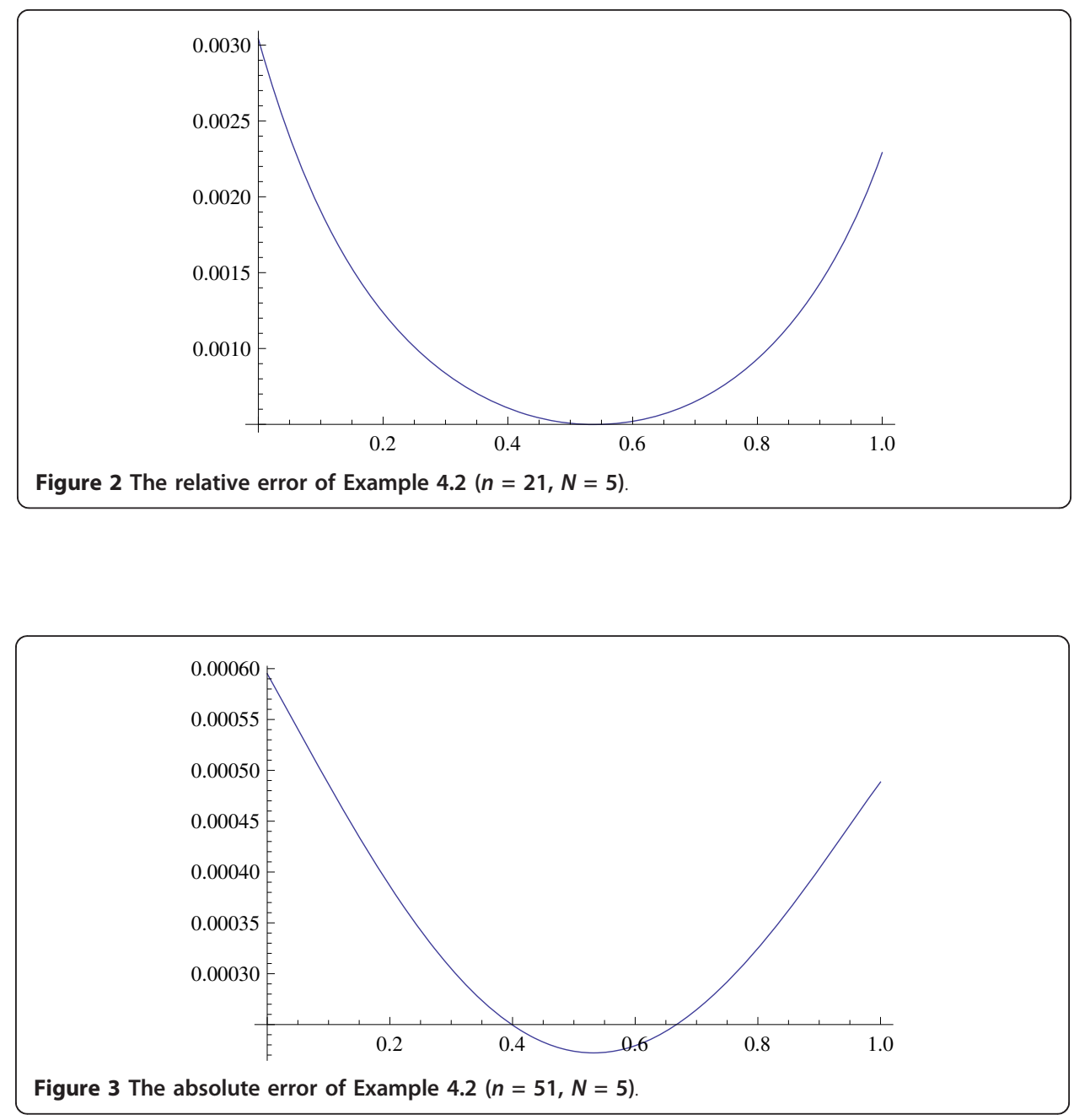

Figure 3 The absolute error of Example $4.2(n=51, N=5)$

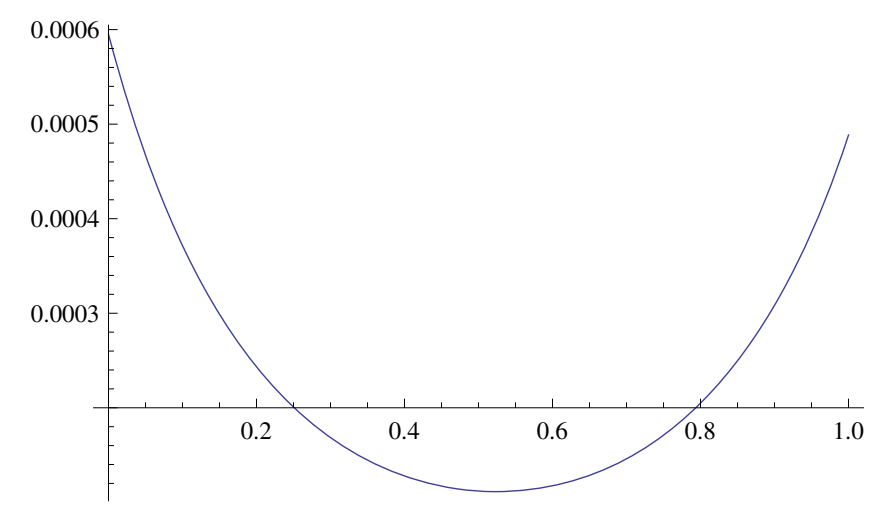

Figure 4 The relative error of Example $4.2(n=51, N=5)$ 


\section{Contributions}

Er Gao gives the main idea and proves the most of the theorems and propositions in the paper. He also takes part in the work of numerical experiment of the main results. Xinjian Zhang suggests some ideas for the prove of the main theorems. Songhe Song mainly accomplishes most part of the numerical experiments. All authors read and approved the final manuscript.

\section{Appendix A: The reproducing kernel of $H_{2}^{3}[0,1]$}

The reproducing kernel of $H_{2}^{3}[0,1]$ is

$$
\begin{aligned}
K_{3}[t, s]=\frac{\Lambda_{1}}{120 \Delta} & +\frac{\Lambda_{2}}{\Delta^{2}}+\frac{\Lambda_{3}}{120 \Delta}+\frac{\Lambda_{4}}{40 \Delta^{2}}+\frac{\Lambda_{5}}{120 \Delta}+\frac{\Lambda_{6}}{120 \Delta}+\frac{\Lambda_{7}}{120 \Delta^{2}} \\
& +\left\{\begin{array}{l}
\frac{1}{120}\left(s^{5}-5 s^{4} t+10 s^{3} t^{2}\right), t \geq s \\
\frac{1}{120}\left(t^{5}-5 t^{4} s+10 t^{3} s^{2}\right), t<s
\end{array}\right.
\end{aligned}
$$

where

$$
\begin{aligned}
& \Delta=a_{3}\left(2 b_{1} b_{2}+b_{2} c_{1}-c_{1} c_{2}\right)+a_{2}\left(a_{3} b_{1}-a_{1} b_{3}+2 a_{1} c_{3}-2 b_{1} c_{3}\right) \\
& -2\left(a_{1}+c_{1}\right)\left(b_{2} b_{3}-b_{2} c_{3}-c_{2} c_{3}\right) \\
& \Lambda_{1}=\left(c _ { 1 } \left(-2 c_{2} c_{3}+a_{3} c_{2} s^{2}-a_{2}(-1+s)\left(b_{3}-2 c_{3}-a_{3} s+b_{3} s\right)\right.\right. \\
& \left.\left.+b_{2}\left(2 b_{3}-2 c_{3}+a_{3}(-2+s) s\right)\right) t^{3}\left(10-5 t+t^{2}\right)\right), \\
& \Lambda_{2}=\left(2 b_{1} b_{2}+b_{2} c_{1}-c_{1} c_{2}-2 a_{1} b_{2} s-2 b_{2} c_{1} s+a_{1} b_{2} s^{2}+b_{2} c_{1} s^{2}\right) \\
& \left.+a_{1} c_{2} s^{2}+c_{1} c_{2} s^{2}-a_{2}(-1+s)\left(b_{1}-a_{1} s+b_{1} s\right)\right) \\
& \times\left(2 b_{1} b_{2}+b_{2} c_{1}-c_{1} c_{2}-2 a_{1} b_{2} t-2 b_{2} c_{1} t+a_{1} b_{2} t^{2}+b_{2} c_{1} t^{2}\right. \\
& \left.+a_{1} c_{2} t^{2}+c_{1} c_{2} t^{3}-a_{2}(-1+t)\left(b_{1}-a_{1} t+b_{1} t\right)\right), \\
& \Lambda_{3}=c_{1} s^{3}\left(10-5 s+s^{2}\right)\left(-a_{2}(-1+t)\left(b_{3}-2 c_{3}-a_{3} t+b_{3} t\right)\right. \\
& -2 c_{2} c_{3}+a_{3} c_{2} t^{2}+b_{2}\left(2 b_{3}-2 c_{3}+a_{3}(-2+t) t\right), \\
& \Lambda_{4}=c_{1}\left(2\left(c_{2} c_{2}\left(-2 c_{3}+a_{3} s^{2}\right)+a_{2}\left(2 b_{1} c_{3}-2 a_{1} c_{3} s-a_{3} b_{1} s^{2}+a_{1} b_{3} s^{2}\right)\right)\right. \\
& +b_{2}\left(10 b_{1} c_{3}+6 c_{1} c_{3}+a_{3} c_{1} s-10 a_{1} c_{3} s-10 c_{1} c_{3} s-5 a_{3} b_{1} s^{2}\right. \\
& \left.\left.-3 a_{3} c_{1} s^{2}+b_{3}\left(-c_{1}+5 a_{1} s^{2}+5 c_{1} s^{2}\right)\right)\right)\left(-2 c_{2} c_{3}+a_{3} c_{s} t^{2}\right. \\
& \left.-a_{2}(-1+t)\left(b_{3}-2 c_{3}-a_{3} t+b_{3} t\right)+b_{2}\left(2 b_{3}-2 c_{3}+a_{3}(-2+t) t\right)\right), \\
& \Lambda_{5}=\left(2 b_{1} c_{3}+2 c_{1} c_{3}+a_{3} c_{1} s-2 a_{1} c_{3} s-2 c_{1} c_{3} s-a_{3} b_{1} s^{2}-a_{3} c_{1} s^{2}\right. \\
& \left.+b_{3}\left(a_{1} s^{2}+c_{1}\left(-1+s^{2}\right)\right)\right) t^{3}\left(-5 b_{2}(-4+t)+a_{2}\left(10-5 t+t^{2}\right)\right), \\
& \Lambda_{6}=s^{3}\left(-5 b_{2}(-4+s)+a_{2}\left(10-5 s+s^{2}\right)\right)\left(2 b_{1} c_{3}+2 c_{1} c_{3}+a_{3} c_{1} t\right. \\
& \left.-2 a_{1} c_{3} t-2 c_{1} c_{3} t-a_{3} b_{1} t^{3}-a_{3} c_{1} t^{2}+b_{3}\left(a_{1} t^{2}+c_{1}\left(-1+t^{2}\right)\right)\right), \\
& \Lambda_{7}=\left(6 a_{2}^{2}\left(a_{1} s\left(-2 c_{3}+b_{3} s\right)+b_{1}\left(2 c_{3}-a_{3} s^{2}\right)\right)+3 a_{2}\left(2 c_{1} c_{2}\left(-2 c_{3}+a_{3} s^{2}\right)\right.\right. \\
& +b_{2}\left(-b_{3} c_{1}+20 b_{1} c_{3}+6 c_{1} c_{3}+a_{3} c_{1} s-20 a_{1} c_{3} s-10 c_{1} c_{3} s-10 a_{3} b_{1} s^{2}\right. \\
& \left.\left.+10 a_{1} b_{3} s^{2}-3 a_{3} c_{1} s^{2}+5 b_{3} c_{1} s^{2}\right)\right)+5 b_{2}\left(3 c_{1} c_{2}\left(-2 c_{3}+a_{3} s^{2}\right)\right. \\
& +b_{2}\left(-2 b_{3} c_{1}+16 b_{1} c_{3}+10 c_{1} c_{3}+2 a_{3} c_{1} s-16 a_{1} c_{3} s-16 c_{1} c_{3} s-8 a_{3} b_{1} s^{2}\right. \\
& \left.\left.\left.+8 a_{1} b_{3} s^{2}-5 a_{3} c_{1} s^{2}+8 b_{3} c_{1} s^{2}\right)\right)\right)\left(2 b_{1} c_{3}+2 c_{1} c_{3}+a_{3} c_{1} t-2 a_{1} c_{3} t\right. \\
& \left.-2 c_{1} c_{3} t-a_{3} b_{1} t^{2}-a_{3} c_{1} t^{2}+b_{3}\left(a_{1} t^{2}+c_{1}\left(-1+t^{2}\right)\right)\right) \text {. }
\end{aligned}
$$


Acknowledgements

The work is supported by NSF of China under Grant Numbers 10971226.

\section{Competing interests}

The authors declare that they have no competing interests.

Received: 13 January 2011 Accepted: 16 January 2012 Published: 16 January 2012

\section{References}

1. Erbe, LH, Wang, HY: On the existence of positive solutions of ordinary differential equations. Proc Am Math Soc. 120(3):743-748 (1994). doi:10.1090/50002-9939-1994-1204373-9

2. Kaufmann, ER, Kosmatov, N: A second-order singular boundary value problem. Comput Math Appl. 47, 1317-1326 (2004). doi:10.1016/S0898-1221(04)90125-3

3. Yang, F-H: Necessary and sufficient condition for the existence of positive solution to a class of singular second-order boundary value problems. Chin J Eng Math. 25(2):281-287 (2008)

4. Zhang, X-G: Positive solutions of nonresonance semipositive singular Dirichlet boundary value problems. Nonlinear Anal. 68, 97-108 (2008). doi:10.1016/.jna.2006.10.034

5. Ma, R-Y, Ma, H-L: Positive solutions for nonlinear discrete periodic boundary value problems. Comput Math Appl. 59, 136-141 (2010). doi:10.1016/j.camwa.2009.07.071

6. Russell, RD, Shampine, LF: A collocation method for boundary value problems. Numer Math. 19, 1-28 (1972). doi:10.1007/BF01395926

7. Stynes, M, O'Riordan, E: A uniformly accurate finite-element method for a singular-perturbation problem in conservative form. SIAM J Nu-mer Anal. 23, 369-375 (1986). doi:10.1137/0723024

8. Xu, JC, Shann, WC: Galerkin-wavelet methods for two-point boundary value problems. Numer Math. 63, 123-144 (1992). doi:10.1007/BF01385851

9. He, J-H: Variational iteration method-a kind of non-linear analytical technique: some examples. Nonlinear Mech. 34 699-708 (1999). doi:10.1016/50020-7462(98)00048-1

10. Capizzano, SS: Spectral behavior of matrix sequences and discretized boundary value problems. Linear Algebra Appl. 337, 37-78 (2001). doi:10.1016/50024-3795(01)00335-4

11. Ilicasu, FO, Schultz, DH: High-order finite-difference techniques for linear singular perturbation boundary value problems. Comput Math Appl. 47, 391-417 (2004). doi:10.1016/S0898-1221(04)90033-8

12. Cui, MG, Geng, F-Z: A computational method for solving one-dimensional variable-coefficient Burgers equation. Appl Math Comput. 188, 1389-1401 (2007). doi:10.1016/j.amc.2006.11.005

13. Cui, MG, Chen, Z: The exact solution of nonlinear age-structured population model. Nonlinear Anal Real World Appl. 8, 1096-1112 (2007). doi:10.1016/j.nonrwa.2006.06.004

14. Geng, FZ: Solving singular second order three-point boundary value problems using reproducing kernel Hilbert space method. Appl Math Comput. 215, 2095-2102 (2009). doi:10.1016/j.amc.2009.08.002

15. Geng, F-Z, Cui, M-G: Solving singular nonlinear second-order periodic boundary value problems in the reproducing kernel space. Appl Math Comput. 192, 389-398 (2007). doi:10.1016/j.amc.2007.03.016

16. Jiang, W, Cui, M-G, Lin, Y-Z: Anti-periodic solutions for Rayleigh-type equations via the reproducing kernel Hilbert space method. Com-mun Nonlinear Sci Numer Simulat. 15, 1754-1758 (2010). doi:10.1016/j.cnsns.2009.07.022

17. Zhang, XJ, Long, H: Computating reproducing kernels for $W_{2}^{m}[a, b]$ (I). Math Numer Sin 30(3):295-304 (2008). (in (hinese)

18. Zhang, XJ, Lu, S-R: Computating reproducing kernels for $W_{2}^{m}[a, b]$ (II). Math Numer Sin 30(4):361-368 (2008). (in (hinese)

19. Long, $\mathrm{H}$, Zhang, $\mathrm{X}$-J: Construction and calculation of reproducing kernel determined by various linear differential operators. Appl Math Comput. 215, 759-766 (2009). doi:10.1016/j.amc.2009.05.063

doi:10.1186/1687-2770-2012-3

Cite this article as: Gao et al.: Solving singular second-orderinitial/boundary value problems in reproducing kernel Hilbert space. Boundary Value Problems 2012 2012:3.

\section{Submit your manuscript to a SpringerOpen ${ }^{\odot}$ journal and benefit from:}

- Convenient online submission

- Rigorous peer review

- Immediate publication on acceptance

- Open access: articles freely available online

- High visibility within the field

- Retaining the copyright to your article

Submit your next manuscript at $\gg$ springeropen.com 